\title{
Study on Regional Water Resources Allocation Efficiency Based on Networking and Joint Scheduling of Reservoir Groups
}

\author{
Ye Zhou \\ Department of Water Conservancy and Environmental Engineering, Zhejiang University of Water Resources and Electric Power, Hangzhou, \\ China
}

\section{Email address: \\ 3043109291@qq.com}

\section{To cite this article:}

Ye Zhou. Study on Regional Water Resources Allocation Efficiency Based on Networking and Joint Scheduling of Reservoir Groups. American Journal of Water Science and Engineering. Vol. 4, No. 2, 2018, pp. 33-39. doi: 10.11648/j.ajwse.20180402.14

Received: June 20, 2018; Accepted: August 6, 2018; Published: August 31, 2018

\begin{abstract}
The effect of reservoir group networking and joint scheduling on the efficiency of regional water resources allocation is studied in the paper and demonstrated by case analysis of two engineering projects in the counties of Zhejiang Province. The efficiency of water resources allocation involves both engineering and economic aspects, and its improvement is the result of the interaction between institutions and technologies. The efficiency of networking and joint scheduling of reservoir groups is obvious, as proved by the physical network created by joint scheduling, the internal incentive mechanism generated by water right transactions, as well as the networking and joint scheduling of agricultural irrigation, urban water supply and ecological water use. This efficiency mainly comes from the accumulation of reservoir resources and the rational utilization of flood resources brought by networking and joint scheduling. It would be an important model for the future development of regional water resources optimization to network and jointly operate reservoir groups. In essence, the networking and joint scheduling of a reservoir group involve the optimal allocation and operation of multi-source water resources for multi-users. The scheduling diagram can be regarded as the combination of diversion and water supply dispatch of each member reservoir, forming the network structure, realizing the joint operation of regional water resources, and finally improving the efficiency of water resources allocation at different stages in different regions. The main body of the reservoir networking and joint scheduling, the government departments, carry out planned dispatching of reservoirs only with obtaining certain amount of information. It is therefore necessary to establish an incentive compatibility mechanism to overcome such defects as information lag and asymmetry, in networking and operating reservoir groups.
\end{abstract}

Keywords: Reservoir Groups, Networking and Joint Scheduling, Regional Water Resources, Allocation Efficiency

\section{Introduction}

Regional water resources allocation is an important part of watershed water resources allocation. The main problem encountered in regional water resources allocation is the uneven distribution of rainfall in time and space. The most important way to solve the problem is to construct reservoirs and carry out scientific scheduling. Before1970s or 1980s, due to the small number of constructed reservoirs, one or two controlling reservoirs were often used to improve the flood control and irrigation capacity of the basin or region, and the efficiency of water resource allocation in the regions without controlling reservoirs was rather low [1]. Since 1990s, with the increasing state investment on water conservancy and other infrastructure facilities, the number of newly built reservoirs in the region has risen steadily, so the joint operation of multiple reservoirs could be carried out in accordance with the situation of water coming from different watersheds in the region, providing an important foundation for improving the efficiency of regional water resources allocation [2].

The allocation of water resources should not only meet the needs of the traditional agricultural irrigation, life and industrial water, but also play a role in the ecological and 
environmental water use. Therefore, the goal of regional water resources allocation is to realize the sustainable development of regional economy and society based on regional water resources endowment while protecting the ecology and environmental security. The scarcer the regional water resources are, the more important is the role of resource allocation [3]. By using modern science and technology, some areas with many reservoirs, or areas with small and medium reservoirs but lack of controlling large reservoirs, can improve the efficiency of water resources allocation through the interconnection and joint operation of reservoirs. The networking and joint scheduling of regional reservoir groups refers to the connection of water reservoirs in a certain area through the construction of tunnels, channels, river courses, etc. to operate them according to the actual water situation. The allocation of regional water resources is thus evolved into the sustainable development of regional economy and society under the condition of ensuring the ecological environment water security [4].

\section{Method}

The study on the regional water resources allocation is done with two typical cases, one in Yongkang, a county-level city, and the other in Ningbo, a prefecture-level city in Zhejiang. In Yongkang, the networking and joint scheduling of reservoirs was initiated in the 1990s mainly for agricultural irrigation, and only after entering the new century did it change from agricultural water use to urban water supply. Whereas in Ningbo, the networking and joint scheduling of reservoirs started only 10 years ago with the initial aim of solving the problem of urban water supply.

\subsection{Yongkang Case: from Irrigation Network to Water Supply Network}

Yongkang is a typical hilly area. There is not a large reservoir in the county, and the largest Yangxi reservoir has a capacity of only $5,316 \mathrm{~m}^{3}$. Before $1990 \mathrm{~s}$, reservoirs were mostly used for agricultural irrigation, and urban water supply was from rivers. Irrigation in the region was particularly difficult due to the rugged land. In order to solve the agricultural irrigation problem, the west-east Huadong Aqueduct of Yangxi Reservoir was utilized to connect the irrigation channels downstream to the four reservoirs in the north of the city, respectively, Hongtangkeng Reservoir, Taiping Reservoir, Shanghuang Reservoir, and Huangfen Reservoir. After the networking, Yongxi and the four reservoirs were jointly operated to make the full utilization of water resources as far as possible. In the late 1990s, the source of water supply in urban area of Yongkang (including Zhiying Community) changed from river water to Yangxi Reservoir. Yangxi Reservoir became the only source of water supply in the area, and since then it has been playing an important role in urban water supply. The actual supply of water to the urban area in 2013 was 49.6 million $\mathrm{m}^{3}$, very close to the designed water supply of 50 million $\mathrm{m}^{3}$ at the guaranteed rate of $95 \%$. However, in recent years, the water demand of urban area has been increasing at a rate of more than $10 \%$ every year. Therefore, the risk of relying on Yangxi Reservoir as the only source of water had been highlighted, and it was necessary to find a new water source for urban water supply. The five reservoirs in the northern part of the city (see Figure 1) have been connected to each other through aqueducts and channels, forming a second source of water for urban water plants (see Table 1).

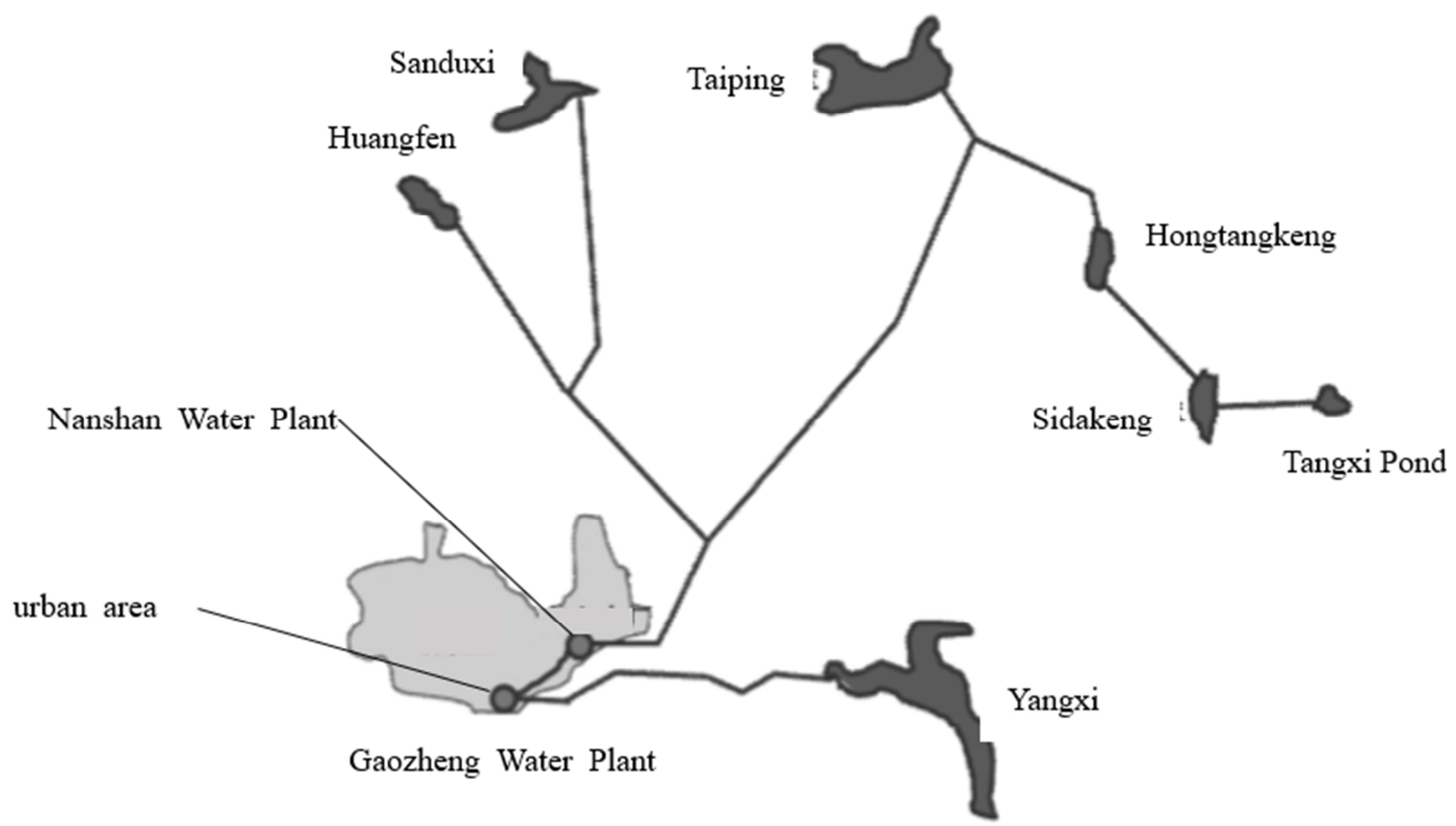

Figure 1. Network and Joint Operation of Reservoirs in Yongkang. 
Table 1. The Related Reservoirs in the Networking Project in Yongkang.

\begin{tabular}{lllllll}
\hline Reservoir & $\begin{array}{l}\text { Catchment } \\
\text { area }\left(\mathbf{k m}^{\mathbf{2}}\right)\end{array}$ & $\begin{array}{l}\text { Normal capacity } \\
\left(\mathbf{1 0} \text { thousand } \mathbf{~ m}^{\mathbf{3}}\right)\end{array}$ & $\begin{array}{l}\text { Normal design } \\
\text { water level } \mathbf{( m )}\end{array}$ & $\begin{array}{l}\text { Water plant and } \\
\text { scale }(\mathbf{1 0 , 0 0 0} \mathbf{t} / \mathbf{d})\end{array}$ & $\begin{array}{l}\text { Population of the water } \\
\text { supply area (10,000)}\end{array}$ & $\begin{array}{l}\text { Design irrigation area } \\
(\mathbf{1 0 , 0 0 0} \mathbf{~ m u})\end{array}$ \\
\hline Yangxi & 124 & 5,316 & 153.84 & Nanshan, 22 & 50 & 14.4 \\
Taiping & 83 & 3,226 & 145.75 & Qiaoxia, 3.75 & 10 & 6.8 \\
Sanduxi & 22.24 & 921 & 198.76 & Xiangzhu, 3.25 & 6 & 3.25 (including Huangfen) \\
Sidakeng & 3.56 & 97 & 262.60 & & & 0.04 \\
Huangfen & 19.93 & 827 & 172.64 & Huangfei, 0.6 & 2 & 2.0 \\
Hongtangkeng & 7.64 & 422 & 172.33 & Hongtangkeng, 0.6 & 2 & 1.0 \\
\hline
\end{tabular}

Note: The scale of Nanshan Water Plant (220,000 t/d) includes that of Zhiying Water Plant (20,000t/d); the catchment area of Huangfen Reservoir is $19.93+$ $9.85 \mathrm{~km}^{2}$ after its expansion, and its normal capacity reaches 8.27 million $\mathrm{m}^{3}$, and normal water level $180 \mathrm{~m}$.

The networking project of the reservoirs in the north of Yongkang consists of three parts. The first part is the newly built Tangxi Pond with new catchment area of $16.9 \mathrm{~km}^{2}$, the continued construction of Sidakeng Reservoir with normal capacity increasing from $348,000 \mathrm{~m}^{3}$ to $970,000 \mathrm{~m}^{3}$, and the expansion of Huangfen Reservoir with normal capacity increasing from $4,350,000 \mathrm{~m}^{3}$ to $8,270,000 \mathrm{~m}^{3}$. The second part is the newly built Tangxi Pond Diversion System to divert the water to Sidakeng Reservoir, increasing a new catchment area of more than $20 \mathrm{~km}^{2}$. The last part is the connection of the five reservoirs in the northern part of the city, namely, Taiping, Hongtangkeng, Sidakeng, Sanduxi and Huangfen, with newly built $7 \mathrm{~km}$-long tunnels and over $40 \mathrm{~km}$-long pipes. As the second water supply source of the city, its annual water supply to the urban area is 30 million $\mathrm{m} 3$, which solves such problems as the single source water supply of Yangxi Reservoir, the shortage of water quantity and the low guarantee rate of water supply. The preliminary cost estimate of the networking project amounts to 820 million yuan.

As can be seen from Table 1, the reservoirs, except Taiping Reservoir, in the reservoir networking project are all small reservoirs. After the implementation of the project, it is estimated that the annual water supply capacity of high-quality water can be increased to about 30 million $\mathrm{m}^{3}$ through the joint operation of the five small and medium-sized reservoirs, equivalent to the construction of a new medium-sized reservoir, which can basically solve the problem of water shortage in the near future. The water demand of forward development could be solved through the diversion project of Qianming Reservoir in the Oujiang River basin and the construction of Pan'an diversion tunnel in Yongkang to divert the water from Qianming Reservoir and Qiuli Reservoir to the city. It is also necessary to establish water right trading and economic compensation mechanism in three related counties and cities.

\subsection{Ningbo Case: from Water Supply Network to Joint Scheduling and Supply}

This case mainly looks at the raw water problem in urban water supply. According to the forecast of Ningbo, the annual water demand of the city will reach and even exceed 3 billion $\mathrm{m}^{3}$ by 2020 , and the high-quality water demand of the central urban area will reach 0.628 billion $\mathrm{m}^{3}$. Currently, the existing reservoirs, including Baixi, Zhougongzhai, Hengshan, Tingxia, Jiaokou, Hengxi, and Sanxipu, can supply 570 million $\mathrm{m}^{3}$ high-quality raw water to the central urban area. When the time comes, the reservoirs in Ningbo will not be able to meet the water demand and it is unlikely to find a place to build a large reservoir. At present, there are 6 large reservoirs, 26 medium-sized reservoirs and 379 small reservoirs in Ningbo. These water sources projects are scattered all over the city with uneven distribution, resulting in abundant water in some places and scarce water in other places. In 2005, a raw water company was established in Ningbo, responsible for the development and management of urban water sources. After the establishment of the company, a cross-basin and cross-regional Qincun Reservoir Water Diversion Project and the West Line Networking and Joint Scheduling Project were successively implemented.

After the implementation of the projects, 11 reservoirs, including Qincun Reservoir, have been scheduled in a unified way. In accordance with the distribution of the reservoir, the reservoir planning of Ningbo categorizes the reservoirs into East Line and West Line and connects them respectively for joint operation. The East Line reservoirs include Baixi, Hengshan, Hengxi, Sanxipu and Dongqianhu (see Table 2), and they are successfully networked. The West Line reservoirs include Tingxia, Zhougongzhai, Jiaokou, Xixia, Qincun (under construction) and Xujiang'an (to be constructed). The networking of the these reservoirs covers four parts, respectively, Qincun Diversion project (from Tingxia to Ningbo), Xixia Diversion Project, the connection project from Jiaokou to Xixia, and the East-West Lines connection project (from Lingjiao to Xiaozheng) (see Figure 2). After the implementation of East-West Lines connection Project, all the 11 reservoirs of East-West Line and East-West Line are connected to each other to realize the joint operation [5]. 


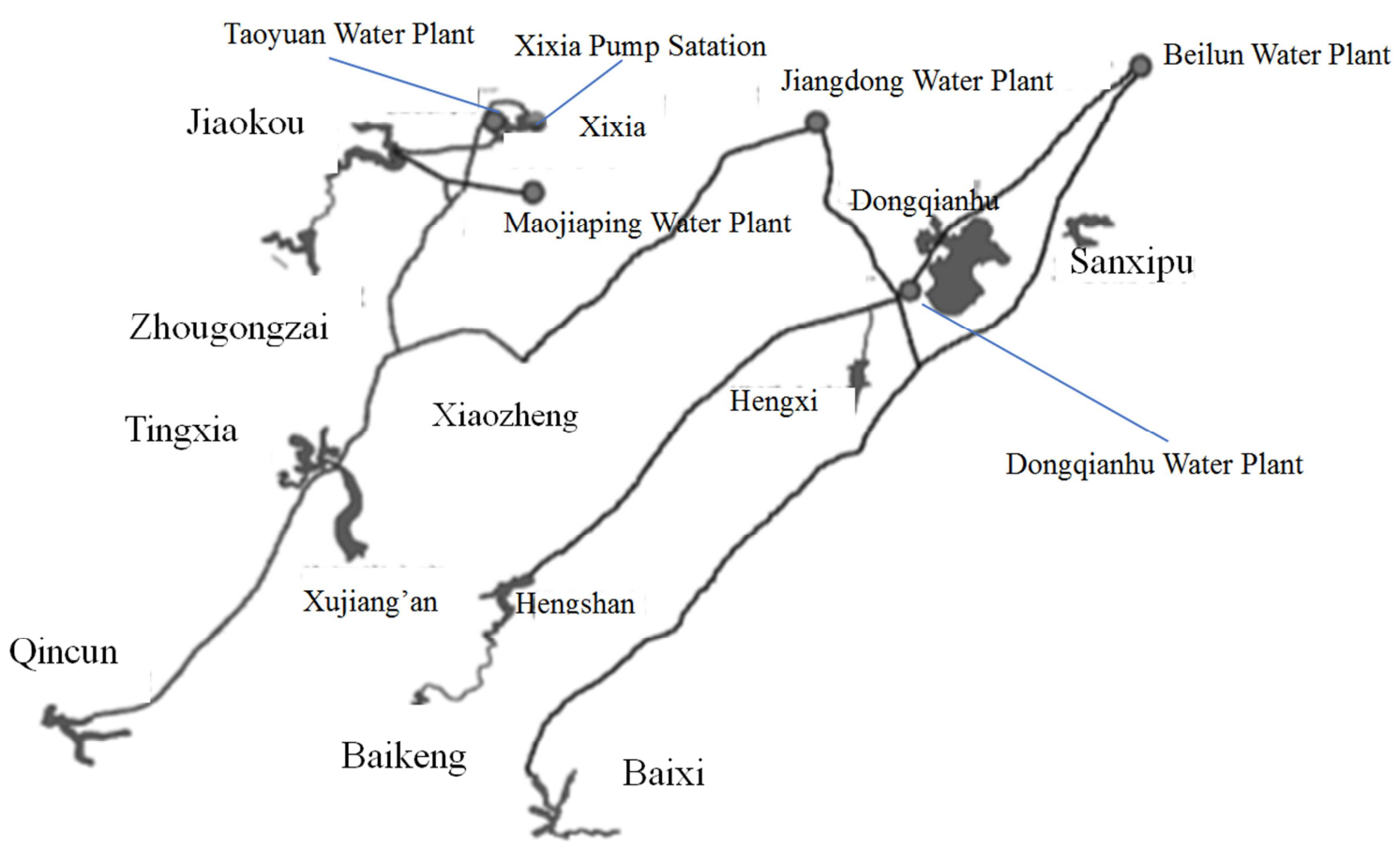

Figure 2. The Networking of Reservoir Groups in Ningbo.

Table 2. Profile of Reservoirs of East Line Network in Ningbo.

\begin{tabular}{|c|c|c|c|c|c|c|}
\hline Reservoir & $\begin{array}{l}\text { Catchment } \\
\text { area }\left(\mathbf{k m}^{2}\right)\end{array}$ & $\begin{array}{l}\text { Normal capacity } \\
\left(100 \text { million } \mathrm{m}^{3}\right)\end{array}$ & $\begin{array}{l}\text { Normal design } \\
\text { water level }(\mathrm{m})\end{array}$ & Water plant & $\begin{array}{l}\text { Population of the water } \\
\text { supply area }(10,000)\end{array}$ & Water plant elevation $(\mathrm{m})$ \\
\hline Baixi & 254 & 1.45 & 170 & $\begin{array}{l}\text { Beilun Jiangdong } \\
\text { Dongqianhu }\end{array}$ & & $\begin{array}{l}2.0 \\
2.3 \\
50\end{array}$ \\
\hline Hengshan & 150.8 & 0.765 & 109.67 & Dongqianhu & & 50 \\
\hline $\begin{array}{l}\text { Hengxi } \\
\text { Dongqianhu }\end{array}$ & 39.8 & 0.25 & 31.42 & & & $\begin{array}{l}\text { For town-level water plant } \\
\text { No water plant }\end{array}$ \\
\hline Sanxipu & 48.8 & 0.245 & 32.62 & $\begin{array}{l}\text { Zhenghai Refinery } \\
\text { Gangpu Co. } \\
\text { Town-level water plant }\end{array}$ & & \\
\hline
\end{tabular}

Table 3. Profile of Reservoirs of West Line Network in Ningbo.

\begin{tabular}{|c|c|c|c|c|c|c|}
\hline Reservoir & $\begin{array}{l}\text { Catchment } \\
\text { area }\left(\mathrm{km}^{2}\right)\end{array}$ & $\begin{array}{l}\text { Normal capacity } \\
\left(100 \text { million } \mathrm{m}^{3}\right)\end{array}$ & $\begin{array}{l}\text { Normal design } \\
\text { water level (m) }\end{array}$ & Water plant $(10,000$ t/d) & $\begin{array}{l}\text { Population of the water } \\
\text { supply area }(10,000)\end{array}$ & $\begin{array}{l}\text { Water plant } \\
\text { elevation }(m)\end{array}$ \\
\hline Qincun & 316 & 1.766 & 98 & Taoyuan (50) (main supply) & & \\
\hline Xixia & 29.9150 .8 & 0.2074 & 55 & Taoyuan (50) (auxiliary supply) & & \\
\hline Tingxia & 176 & 1.00 & 82.65 & Jiangdong (joint supply) & & 2.3 \\
\hline $\begin{array}{l}\text { Xujiang'an } \\
\text { (planning) }\end{array}$ & & & & Jiangdong (joint supply) & & \\
\hline Jiaokou & 259 & 0.7807 & 68.08 & Maijiaping (50) & & \\
\hline Zhougongzhai & 132 & 0.9608 & 231.13 & Maijiaping (50) & & \\
\hline
\end{tabular}

Note: Taoyuan Water Plant was built in 2017 with design water supply capacity of 500,000 t/d, mainly for Hangzhou Bay New Area; Tingxia Reservoir is connected to the network while ensuring the water supply of Fenghua and downstream areas.

After the connection project was completed, the original pattern of original water supply by corresponding reservoirs was broken, and a new pattern of network water supply was formed. For example, originally, Jiaokou Reservoir and Zhougongzhai Reservoir supplied water for Maojiaping Water Plant; Qincun Reservoir and Ting Xia Reservoir supplied water for Taoyuan Water Plant; Baixi Reservoir and Hengshan Reservoir supplied water for Beilun Water Plant, Jiangdong Water Plant and Dongqianhu Water plant. Now, these water plants are supplied by the reservoir network formed by the connection project.

\section{Result}

In these two cases with different networking time, regional scale and service target, we can find that the networking and joint scheduling of reservoir groups might show different efficiency at different stages and different regions. The characteristics of Yongkang case are as follows: first, through the adjustment of the industrial structure, the use of water 
resources is transferred from agricultural purpose to urban water consumption; second, the water resources of several small reservoirs can be concentrated and efficiently utilized by the networking of reservoirs, providing a second water source for urban area with the joint operation of reservoir network; third, the further increase of water supply in the future needs to be solved through the external diversion of water, and the exchange of water rights to form an incentive mechanism. Ningbo case is featured as follows: first, several large and medium-sized reservoirs are connected to form a water supply network, improving the guarantee rate of water supply; second, the supply of water resources is increased by about 37 million $\mathrm{m}^{3}$ per year through the utilization of resources during the flood season; third, dual water supply is realized, where the connected reservoirs are responsible for living and environmental water use, while the other reservoirs are responsible for industrial water use.

The comparative study of these two cases shows that from the perspective of resource integration, the market mechanism, as represented by Ningbo, allows more room for networking and joint scheduling of water resources than the mandatory planning mechanism, as represented by Yongkang, does. Moreover, the very big difference in the benefits of reservoir network connection and joint operation caused by the different water resources quantity indicates that the large and medium-sized reservoirs, as those in Ningbo, are more suitable for larger investments than the medium and small reservoirs, as those in Yongkang, are. Additionally, the study finds that the water resources in Yongkang are jointly scheduled under the single county government, while in Ningbo, the joint scheduling of the reservoirs involves the interests of the city, several counties under it, and Xinchang, the county not under its jurisdiction as well, which results in different coordination efficiency. The coordination efficiency of Yongkang case is obviously higher than that of Ningbo case.

\section{Discussion}

\subsection{Networking and Joint Scheduling Project of Reservoir Groups}

The reservoir groups networking and joint scheduling project consists of three parts. One is the connection of reservoir groups by channels, penstocks and tunnels to form a reservoir network. The second part is the joint water regulation. When the precipitation in different river basins varies greatly, the amount of water can be adjusted between reservoirs in different flow regions. Especially during the flood period, the surplus flood can be adjusted to other reservoirs with sufficient reservoir capacity to reduce the abandonment of water. The third if the joint water supply. With the network of reservoir groups, water can be supplied to the region for all kinds of uses, including industrial and agricultural water use, urban domestic water use, river landscape water use, ecological water use, etc. This kind of water supply can be a combination of many schemes [6].

\subsection{The Mechanism of Improving Resources Allocation Efficiency by Networking and Joint Scheduling}

Water resource is a kind of resource, and reservoir capacity is also a kind of resource for water resources. The utilization efficiency of these two resources can be fully played through the networking and joint scheduling of reservoir groups so as to finally improve the efficiency of utilizing water resources [7]. Generally speaking, this efficiency improvement brought by the networking and joint scheduling of reservoir groups is mainly reflected in the following three aspects.

Firstly, the use of water resources can be increased through networking. Especially for the region with many small reservoirs, the scattered small resources can be used intensively as a large water source after networking, and the value of water resource utilization can be enhanced. Also, with the incentive water right trade, surplus agricultural water and environmental water can be effectively converted to water for domestic and industrial uses [8].

Secondly, the reduction of reservoir abandonment, realized by the networking and joint scheduling of reservoir groups, can improve the water resource utilization rate. Compared with the scattered reservoirs, the combined reservoir groups contribute to the system efficiency in the ways as follows: i) under the condition of the same rainfall, the runoff of the reservoir with large catchment area can be adjusted to the reservoir with small rainwater catchment area through the combined regulation; ii) the incoordination between large reservoirs and small water plants, or small reservoirs and large water plants, due to either geographical locations or economic structure, can be solved through the network connection of reservoirs; and iii) the risks that the water supply enterprises are faced with can be reduced. The water supply network can adjust the amount of water supply between different regions, and reduce the risk of water demand fluctuation for water plants, especially the risk of water demand fluctuation brought by the overall rise and fall of some industries [9].

Lastly, networking and joint scheduling of reservoir groups can improve the water supply guarantee rate in the event of an emergency. Particularly, the ecological benefits will be highlighted in case of such emergency event as pollution, as the ecological flow and environmental water quantity of the river in the region can be ensured by reservoir connection and joint regulation.

\subsection{Establishing Incentive Market Mechanism by Introducing Water Rights Trade}

The government departments responsible for the networking and joint scheduling of reservoir groups regulate the reservoirs as planned under the condition of obtaining certain information. It is therefore necessary to establish an incentive compatible mechanism to overcome some defects, such as information lag and asymmetry, in reservoir network connection and operation [10].

Theoretically, it is possible that the network of reservoirs realizes the joint operation of regional water resources, and 
finally improves the efficiency of water resources allocation. However, due to the uncertainty of rainfall and the public attribute of water supply, the realization of the expected efficiency improvement must be based on solving the problem of institutional mechanism [11]. From the perspective of mechanism, it is necessary to establish a water right trading market so that all reservoirs and water users have internal economic incentives and make public the information of water resources scarcity [12]. At the same time, the government intervention mechanism should be set up to directly intervene in the transfer of water between reservoirs when the severe drought disaster occurs, so as to eliminate all kinds of worries of the players in the water right transaction [13]. From the perspective of institution, reservoir administrators, water supply enterprises, irrigation enterprise should be independent stakeholders pursuing the benefit maximization. These independent stakeholders are the precondition of implementing water right transaction, as well as the precondition of water resource allocation efficiency [14].

The efficiency of water resources allocation comes from the self-interest behavior of the stakeholders and is the result of internal motivation. The water resources in the reservoir can be used mainly for agricultural, living, industrial, environmental and ecological purposes. Water for living (industrial) uses goes into the penstock, and the rest of the water is either stored in the river or transported through it. When the water in the river is diverted to the penstock, the purpose of utilizing water resources and the stakeholders of them would change as the marginal value turns from the low end to the higher end. If this change is accompanied by transactions and corresponding economic compensations, it is a kind of sustainable development behavior where each stakeholder has the intrinsic motive for transactions which can be called a kind of incentive compatibility.

\section{Conclusion}

The efficiency of water resources allocation has been improved by the networking and joint scheduling of regional reservoirs mainly in three ways. The first is the progress of engineering technology, especially the application of network flow theory, has enabled many reservoirs to connect with each other through pipes, tunnels and canals into a network to realize joint scheduling. Secondly, the administrative instruction of the government with its planning attribute has been utilized to reduce the transactional cost and improves the efficiency of water right coordination. Thirdly, the incentive compatibility system has been established through the effective use of the market mechanism with stakeholders negotiating with each other and the price mechanism as well, and the efficiency of water resources utilization has been improved. It proves once again the important role of the market and the government in the allocation of water resources. The water right transaction among reservoirs in the region can achieve the compatibility of incentives. More broadly, in the cross-region water diversion, water rights trading and economic compensation can also be utilized to effectively deal with the distribution of interests among different stakeholders and reduce transaction costs so as to improve resource allocation efficiency. Therefore, the property right transaction and cooperative game under the system guarantee are very important in the joint scheduling either through the integration of water resources in the region or the cross-region water diversion.

\section{References}

[1] Huang, Danlu; Ma, Yiming; Xu, Dongmei; et al. Study on Optimal Operation of Reservoirs Combined with Flood Control. Energy and Environment, 2017, 39 (1):65-70.

[2] Wan, Xinyu; Guang, Xiufeng; Zhong, Ping'an; et al. Impact Analysis of Large Reservoir Groups on Watershed Flood Process. Advances in Science and Technology of Water Resources, 2017, 37 (3):66-71.

[3] Yang, Wangwang; Bai, Tao; Ha, Yanping; et al. Study on the Joint Operation of Cascade Reservoirs in the Upper Reaches of Hanjiang River. Journal of Water Resources Research, 2017 (4):340-348.

[4] Wang, Fangfang; Lei, Xiaohui; Peng, Yong; et al. Study on Emergency Flood Control Operation of Xijiang Reservoir Group with Consideration of Hydropower Regulation and Storage. China Rural Water and Hydropower, 2017 (4):189-193.

[5] Zhou, Fen; Zhong, Mingjun; Huang, Rifang. Simulation Study on Water Supply Network System of Reservoir Group in the West of Ningbo. Zhejiang Hydrotechnics, 2015, 43 (6):19-22.

[6] Peng, Yong; Xu, Wei; Jiang, Hongguang. Study on Joint Operation of Urban Water Supply Reservoir Group in the West of Shenzhen. Journal of Hydraulic Engineering, 2016, 35 (11):74-83.

[7] Wu, Hengqing; Huang, Qiang; Xu, Wei; et al. Multi-objective Optimal Scheduling of Reservoir Water Diversion and Supply Based on Aggregation Model. Transactions of the Chinese Society of Agricultural Engineering, 2016, 32 (1):140-146.

[8] Chen, Jin. Water Resources Regulation and Reservoir Group Regulation in the Yangtze River Basi. Journal of Hydraulic Engineering, 2018, 49 (1):2-8.

[9] Chen, Yangbo; Hu, Jiaqi. Study on the Joint Operation Scheme of Cascade Reservoirs in Geheyan and Gaobazhou. Journal of Hydraulic Engineering, 2004 (3):47-52.

[10] Ha, Yanping; Bai, Tao; Huang, Qiang; et al. Study on Multi-objective Transformation of Joint Operation of Water and Sediment in Cascade Reservoirs. Journal of Hydroelectric Engineering, 2017, 36 (7):23-33.

[11] Fang, Hongbin; Wang, Liang; Li, Xinjie. Research Progress of Reservoir Group Operating Rules. Hydrology, 2017, 37 (1):14-18.

[12] Wu, Zhen; Gu, Wenquan; Shao, Dongguo. Study on Optimal Operation of Multiple Reservoirs in Irrigation Area. China Rural Water and Hydropower, 2016 (8):25-27. 
[13] Xu, Zengpei; Li, Hongjun; Wang, Jingjing; et al. Analytical Method for Joint Operation of Parallel Reservoirs in Urban Flood Control Planning. China Rural Water and Hydropower, 2017 (11):119-123.
[14] Ye, Zhou. Research on Incentive Compatibility System in Regional Water Resources Optimal Allocation. Small Hydro Power. 2018 (3):5-9.

\section{Biography}

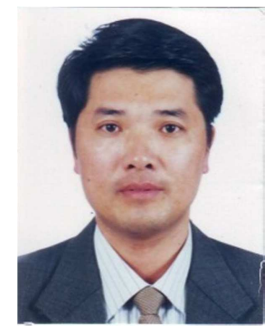

Ye Zhou, graduated from Hehai University in 1986 with bachelor's degree in hydraulic engineer, $\mathrm{PhD}$ in economics of Zhejiang University and professor of engineering, has long been engaging in the study on water resources and hydropower resources. He published more than 40 papers, completed nearly 20 research projects, and published two book. His research and practice of the Three-compensation Mechanism for the Paid Use of Hydropower Resources won the second prize of Dayu Water Science and Technology Award of the Ministry of Water Resources in December 2014. 